host was actually in a long-term cold environment may well have favorable bioenergetic effects in overweight individuals.

\section{Acknowledgments}

Andrew J. Whittle and Antonio VidalPuig are supported by the Biotechnology and Biological Sciences Research Council (BBSRC), the Medical Research Council (MRC), Wellcome Trust, and the European Commission (FP7 BetaBAT).

Address correspondence to: Antonio VidalPuig, University of Cambridge Metabolic Research Laboratories, Level 4, Institute of Metabolic Science, Box 289, Addenbrooke's Hospital, Cambridge CB2 0QQ, United Kingdom. Phone: 44.1223.762790; Fax: 44.1223.330598; E-mail: ajv22@cam.ac.uk.

1. Cypess AM, et al. Identification and importance of brown adipose tissue in adult humans. $N$ Engl J Med. 2009;360(15):1509-1517.

2. Ouellet V, et al. Outdoor temperature, age, sex, body mass index, and diabetic status determine the prevalence, mass, and glucose-uptake activity of 18F-FDG-detected BAT in humans. J Clin Endocrinol Metab. 2011;96(1):192-199.
3. Saito M, et al. High incidence of metabolically active brown adipose tissue in healthy adult humans: effects of cold exposure and adiposity. Diabetes. 2009;58(7):1526-1531.

4. Cannon B, Nedergaard J. Brown adipose tissue: function and physiological significance. Physiol Rev. 2004;84(1):277-359.

5. Seale $P$, et al. PRDM16 controls a brown fat/skeletal muscle switch. Nature. 2008;454(7207):961-967.

6 . Bordicchia $M$, et al. Cardiac natriuretic peptides act via p38 MAPK to induce the brown fat thermogenic program in mouse and human adipocytes. J Clin Invest. 2012;122(3):1022-1036.

7. Cao W, et al. p38 mitogen-activated protein kinase is the central regulator of cyclic AMP-dependent transcription of the brown fat uncoupling protein 1 gene. Mol Cell Biol. 2004;24(7):3057-3067.

8. Cao W, Medvedev AV, Daniel KW, Collins S. betaAdrenergic activation of $\mathrm{p} 38 \mathrm{MAP}$ kinase in adipocytes: cAMP induction of the uncoupling protein 1 (UCP1) gene requires p38 MAP kinase.J Biol Chem. 2001;276(29):27077-27082.

9. Astrup A, Lundsgaard C, Madsen J, Christensen NJ. Enhanced thermogenic responsiveness during chronic ephedrine treatment in man. Am J Clin Nutr. 1985;42(1):83-94

10. Finer N, Bloom SR, Frost GS, Banks LM, Griffiths $\mathrm{J}$. Sibutramine is effective for weight loss and diabetic control in obesity with type 2 diabetes: a randomised, double-blind, placebo-controlled study. Diabetes Obes Metab. 2000;2(2):105-112.

11. Haller CA, Benowitz NL. Adverse cardiovascular and central nervous system events associated with dietary supplements containing ephedra alkaloids.
N Engl J Med. 2000;343(25):1833-1838.

12. Torp-Pedersen $\mathrm{C}$, et al. Cardiovascular responses to weight management and sibutramine in highrisk subjects: an analysis from the SCOUT trial. Eur Heart J. 2007;28(23):2915-2923.

13. Misono KS. Natriuretic peptides and their receptors. FEBS J. 2011;278(11):1791.

14. Hondares E, Rosell M, Gonzalez FJ, Giralt M, Iglesias R, Villarroya F. Hepatic FGF21 expression is induced at birth via PPARalpha in response to milk intake and contributes to thermogenic activation of neonatal brown fat. Cell Metab. 2010; 11(3):206-212.

15. Ogawa Y, et al. BetaKlotho is required for metabolic activity of fibroblast growth factor 21. Proc Natl Acad Sci U S A. 2007;104(18):7432-7437.

16. Sellayah D, Bharaj P, Sikder D. Orexin is required for brown adipose tissue development, differentiation, and function. Cell Metab. 2011;14(4):478-490.

17. Tseng YH, et al. New role of bone morphogenetic protein 7 in brown adipogenesis and energy expenditure. Nature. 2008;454(7207):1000-1004.

18. Watanabe $\mathrm{M}$, et al. Bile acids induce energy expenditure by promoting intracellular thyroid hormone activation. Nature. 2006;439(7075):484-489.

19. Petrovic V, Buzadzic B, Korac A, Vasilijevic A Jankovic A, Korac B. NO modulates the molecular basis of rat interscapular brown adipose tissue thermogenesis. Comp Biochem Physiol C Toxicol Pharmacol. 2010;152(2):147-159.

20. Rothwell NJ, Stock MJ. Influence of environmental temperature on energy balance, diet-induced thermogenesis and brown fat activity in 'cafeteria'-fed rats. BrJ Nutr. 1986;56(1):123-129.

\title{
DYRK1A in Down syndrome: an oncogene or tumor suppressor?
}

\author{
Yehudit Birger ${ }^{1}$ and Shai Izraeli1,2 \\ ${ }^{1}$ Functional Genomics and Department of Pediatric Hemato-oncology, Edmond and Lily Safra Children's Hospital, Sheba Medical Center, \\ Tel Hashomer, Ramat Gan, Israel. ${ }^{2}$ Sackler Faculty of Medicine, Tel Aviv University, Tel Aviv, Israel.
}

\begin{abstract}
Children with Down syndrome (DS) have a markedly increased risk of developing acute megakaryoblastic leukemia (AMKL) and acute lymphoblastic leukemia compared with that of children without DS. Despite recent breakthroughs, it is not clear which genes on chromosome 21, the chromosome that is trisomic in individuals with DS, cause this predisposition. In this issue of the JCI, Malinge et al. report their loss- and gain-of-function experiments in mouse and human cells that show that increased expression of the kinase encoded by the chromosome 21 gene DYRK1A suppresses the nuclear factor of activated $T$ cells pathway and promotes AMKL. Interestingly, the same protein has been suggested to contribute to the reduced risk of epithelial cancers in adults with DS, leading to the possibility that it could be proleukemic in children and antitumorigenic in adults.
\end{abstract}

Down syndrome (DS) is one of the most common genetic birth defects, affecting about 1 in 800 newborns a year in the

Conflict of interest: The authors have declared that no conflict of interest exists.

Citation for this article: J Clin Invest. 2012; 122(3):807-810. doi:10.1172/JCI62372.
United States. It is caused by constitutional trisomy 21 (cT21). The symptoms of DS are heterogeneous, ranging from mild to severe, as are the associated medical conditions. One of the associated medical conditions is leukemia - children with DS have a markedly higher risk of developing both acute lymphoblastic leukemia (ALL) and acute myeloid leukemia than do children who do not have DS. This is in sharp contrast with the reduced prevalence of epithelial cancers in adult individuals with DS (1). Thus, cT21 is proleukemogenic during the pediatric period but antitumorigenic in adulthood. In this issue of the JCI, Malinge et al. provide new insight into the functional contribution of trisomy 21 to leukemogenesis that may have therapeutic implications (2).

\section{DS-associated myeloid leukemias and the role of $\mathrm{cT} 21$}

DS-associated myeloid leukemias evolve in a unique, multistep clinical pattern (Figure 1 and reviewed in ref. 3). Epidemiologic studies have indicated that five percent of newborns with DS have transient myeloproliferative disorder (TMD), a condition that is characterized by the clonal expansion of immature megakaryo- 


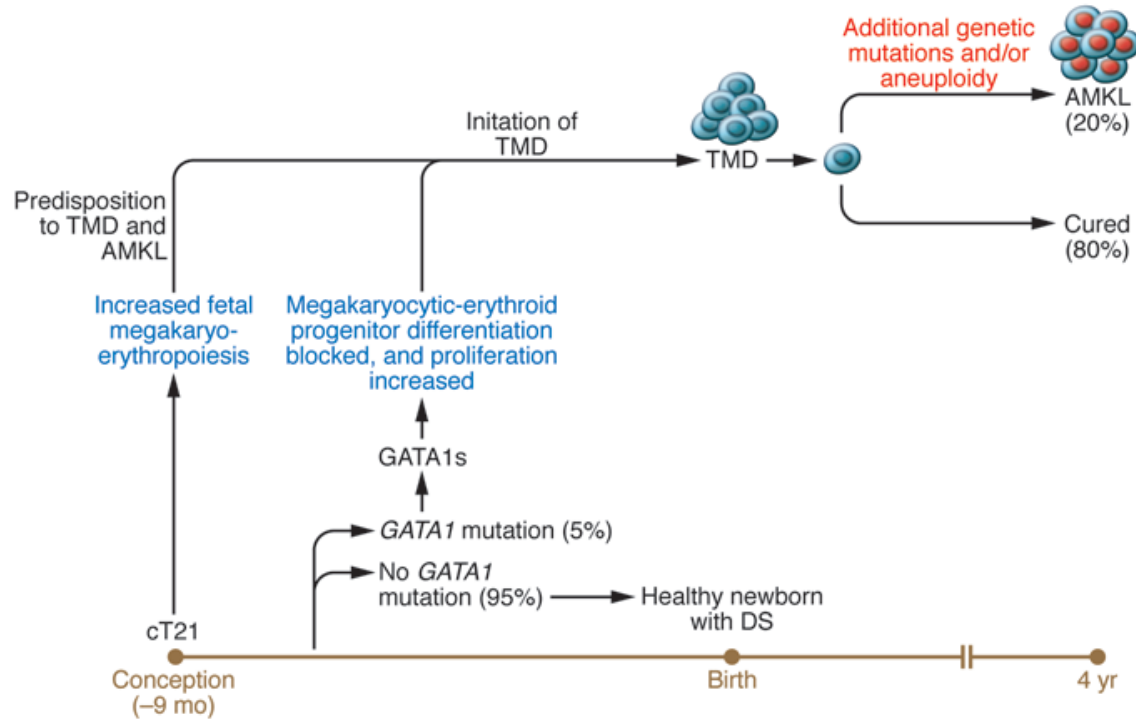

Figure 1

Multistep pathogenesis of the megakaryocytic malignancies of DS. cT21 promotes cell-autonomous fetal megakaryo-erythropoiesis. Acquired mutation of GATA1, leading to expression of GATA1s, further enhances the proliferation of these precursors and blocks terminal megakaryocytic proliferation, manifesting at birth as TMD. TMD is transient; however, in $20 \%$ of the patients, a residual submicroscopic clone acquires additional genetic abnormalities, examples of which are discussed in the main text, that lead to AMKL, which is always diagnosed before 4 years of age.

blasts and is indistinguishable from acute leukemia. The majority of infants survive, and the condition resolves spontaneously by 3 months of age. However, about $20 \%$ of newborns diagnosed with TMD go on to develop acute megakaryoblastic leukemia (AMKL) before 4 years of age. Fortunately, the majority of these patients can be cured with chemotherapy.

Virtually all the TMD and AMKL cells isolated from individuals with DS have been found to carry an acquired mutation in the gene on chromosome $\mathrm{X}$ that encodes the transcription factor GATA1. While mutations differ among patients, and occasionally more than one mutation is identified in the same patient, in all instances they result in the generation of a short GATA1 protein lacking the $\mathrm{N}$-terminal domain (GATA1s). GATA1 is a critical regulator of the megakaryocytic and erythroid lineage, and, in the presence of GATA1s, megakaryocytic differentiation is impaired and the proliferation of fetal megakaryocytic precursors is enhanced, possibly via activation of the transcription factor E2F. Progression of TMD to AMKL is associated with the acquisition of heterogeneous additional mutations in genes such as JAK2,JAK3, MPL, and $P 53$, and additional chromosomal aberrations, resulting in aneuploidy.
The role of CT21 in promoting TMD and AMKL was a mystery until the publication of two independent breakthrough studies in $2008(4,5)$. Both teams of researchers analyzed fetal livers from human fetuses with DS and discovered a dramatic cellautonomous increase in the frequency of megakaryocytic-erythroid progenitors. Importantly, this abnormality preceded the acquisition of the GATA1 mutation. Thus, cT21 provides developmental pressure toward the megakaryocytic-erythroid lineage and constitutes a first "hit" that predisposes all fetuses with DS to TMD and AMKL. In $5 \%$ of the fetuses, this synergizes with the effects of a second hit, the subsequently acquired GATA1 mutations. The GATA1s proteins encoded by the acquired GATA1 mutations block the differentiation of megakaryocytic-erythroid progenitors and enhance the proliferation of these cells to cause congenital TMD (ref. 6 and Figure 1). Finally, in $20 \%$ of the children with DS with TMD, one or more of several different third hits causes progression to AMKL.

Despite this breakthrough, it was not clear which of the genes on chromosome 21 promoted the development of TMD and AMKL. As trisomy results in a small increase in the expression of multiple genes, rather than a marked increase in the expression of one gene, as occurs as a result of single gene amplification (7), it is plausible that the products of several genes on chromosome 21 cooperate in this process. The strongest candidates identified thus far, with varying degrees of experimental evidence, are RUNX1 (8) and ERG (9-11), while an additional study has implicated the chromosome 21-encoded microRNA 125-b2 (12).

In this issue of the JCI, Malinge et al. report their identification of four chromosome 21 genes as candidate mediators of the effect of cT21 on myeloid leukemogenesis. Combining an shRNA screen of human AMKL cell lines with detailed analysis of gene expression in leukemic cells from patients with AMKL with and without DS and functional studies in a mouse model of DS-AMKL that they created, Malinge et al. found evidence that the chromosome 21 genes ERG, DYRK1A, CHAF1B, and HLCS could promote myeloid leukemogenesis. They focused their functional analyses on DYRK1A, which encodes dual-specificity tyrosine-(Y)-phosphorylation-regulated kinase 1A. Unlike ERG, which is involved in leukemias in patients with and without DS, the data suggest that DYRK1A is associated specifically with AMKL in individuals with cT21.

\section{DYRK1A, nuclear factor of activated T cells, and DS}

DYRK1A has multiple substrates (13), but its best-characterized targets, especially in relation to DS, are the five closely related nuclear factor of activated T cells (NFAT) transcriptional regulators, NFAT1-NFAT5 (14). As their name suggests, the NFAT proteins were originally discovered in $\mathrm{T}$ cells and shown to regulate $\mathrm{T}$ cell activation. Since then, it has become clear that they are involved in numerous developmental programs, involving many tissues outside the immune system. In general, activation of the NFAT pathway is considered to be cancer promoting through several possible mechanisms (15). In T lymphoid malignancies, the NFAT pathway is thought to enhance the proliferation and survival of leukemic cells as a result of its fundamental role in $\mathrm{T}$ cell activation. A second mechanism relevant for most solid tumors is that activation of the NFAT pathway can enhance angiogenesis, as transcription of $V E G F$ is increased by NFAT proteins. NFAT proteins also enhance metastatic dissemination and have recently been shown to regulate epithelial-to-mesenchymal transition, which is important for both lineage 


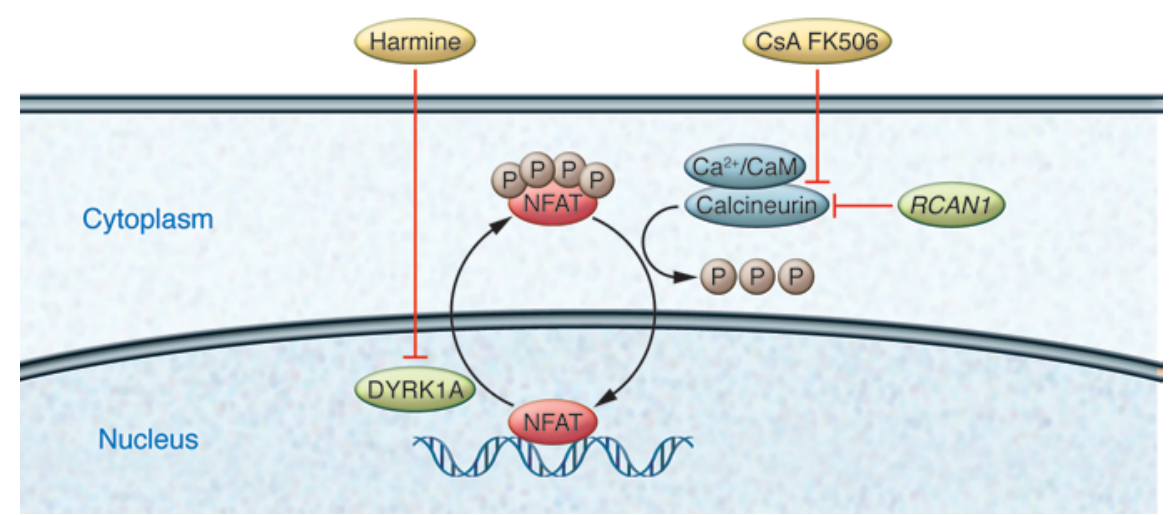

Figure 2

A simplified scheme of the effect of chromosome 21-encoded gene products on the NFAT pathway. The phosphorylation status of NFAT proteins determines their subcellular localization and consequently their transcriptional activity. The phosphatase calcineurin, which is activated by $\mathrm{Ca}^{2+}$ bound to calmodulin $\left(\mathrm{Ca}^{2+} / \mathrm{CaM}\right)$, dephosphorylates NFAT proteins, leading to their translocation to the nucleus. Calcineurin activity is inhibited by calcipressin 1, which is encoded by the chromosome 21 gene RCAN1. In the nucleus, NFAT (in a complex with other transcription factors) regulates the expression of multiple genes. DYRK1A phosphorylates NFAT proteins in the nucleus and facilitates their phosphorylation by additional kinases (GSK3 and CKI). Phosphorylated NFAT proteins are inactivated by translocation to the cytosol. Cyclosporine A (CsA) and FK506 are pharmacological inhibitors of the pathway, acting by blocking calcineurin. Pharmacological inhibitors of DYRK1A, such as harmine, activate the NFAT pathway.

specification during development and metastatic spread of epithelial cancers (16).

The NFAT proteins are the final messengers in a vertebrate-specific signaling cascade initiated by an increase in the concentration of intracellular $\mathrm{Ca}^{2+}$ in response to various external stimuli (e.g., $\mathrm{T}$ cell activation) (Figure 2 and ref. 15). The subcellular localization of NFAT proteins depends on their phosphorylation status. Phosphorylated NFAT proteins are cytosolic and hence transcriptionally inactive. $\mathrm{Ca}^{2+}$ binds to the calcium sensor calmodulin, which in turn binds and activates the protein phosphatase calcineurin. After dephosphorylation by calcineurin, NFAT proteins translocate to the nucleus, in which they cooperate with multiple transcription factors to regulate gene expression. Nuclear NFAT proteins are phosphorylated by DYRK1A, glycogen synthase kinase-3 (GSK3), and casein kinase 1 (CKI) and translocate back to the cytosol. DYRK1A is a "priming" kinase, as phosphorylation mediated by DYRK1A is required for subsequent NFAT phosphorylation by GSK3.

DYRK1A is one of two inhibitors of the NFAT pathway encoded by genes in the "DS-critical region" of the long arm of chromosome 21. The other is calcipressin 1 , which binds and inhibits calcineurin and is encoded by RCAN1 (also known as
DSCR1), itself a target of NFAT. Consistent with this, experimental and mathematical evidence suggest that the NFAT pathway is inhibited in cells from individuals with DS (14). Moreover, a pathologic effect of this inhibition is suggested by the fact that mice lacking NFAT factors or overexpressing DYRK1A have skeletal, neuronal, behavioral, and cardiac defects reminiscent of those observed in patients with DS. Individuals with DS also have impaired thymic function associated with immunodeficiency $(17,18)$. Interestingly, although children with DS have a markedly increased risk of $B$ cell precursor ALL, T-ALL is extremely rare in individuals with DS (3). One may speculate that both the immunodeficiency and the rarity of T-ALL may be also related to inhibition of the NFAT pathway in DS.

Suppression of the NFAT pathway by the chromosome 21-encoded proteins DYRK1A and RCAN1 has also been proposed to explain the lower rate of invasive epithelial cancers in adults with DS (19). Paradoxically, the data generated by Malinge et al. (2) suggest that inhibition of NFAT proteins by DYRK1A promotes the megakaryocytic malignancies associated with DS. It is worthwhile to note that these investigators mainly showed that inhibition of the NFAT pathway correlated with overexpression of DYRK1A and therefore did not rule out the possibility that other substrates may mediate the leuke- mogenic activity of DYRK1A. Interestingly, another inhibitor of calcineurin, FKBP51, has been shown to be induced during megakaryocytic differentiation and to accumulate in idiopathic myelofibrosis, a megakaryocytic neoplasm (20). These independent observations provide strong support for the hypothesis that NFAT inhibition and megakaryocytic malignancies are linked.

The data generated by Malinge et al. (2) support the hypothesis that, while DYRK1A suppresses the growth of epithelial and lymphoid tumors, it is a megakaryocytic oncogene. A similar dual role has been proposed for another chromosome 21-encoded protein, RUNX1. RUNX1 is a tumor suppressor in both lymphoid and myeloid leukemias but has been suggested to be involved, as a result of its overexpression, in DS-AMKL (8).

\section{Future perspective and challenges}

Despite the progress made by Malinge et al. (2) and other researchers $(8-10,11,12,21)$ in deciphering single candidate genes that mediate the leukemogenic effect of cT21, it is still unclear how cT21 functions during the transition from preleukemia to TMD. How many genes on chromosome 21 participate in the leukemogenesis process, and what is the quantitative contribution of each gene? At what stage of development are they involved? What is the basis for the unique and specific cooperation between cT21 and the GATA1 mutation?

Chromosomal aneuploidies are commonly detected in cancer (22). Thus, the answers to these questions as well as additional studies of the role of cT21 in the leukemias associated with DS may have general implications for understanding the oncogenic mechanisms of acquired chromosomal aneuploidies.

A major experimental hurdle has been the lack of appropriate experimental models. Although Malinge et al. have nicely shown that DS-AMKL can be modeled in the mouse by introducing three hits, namely cT21, Gata1 mutation, and a mutation in $M p l$ (2), mouse models of DS are limited. For example, they do not have the fetal phenotype observed in human DS fetuses. DSAMKL cell lines might not be appropriate for study, as they represent a stage occurring after either the preleukemic or TMD phase. Substantial progress has been made recently by the expansion of trisomic hematopoietic cells from DS fetuses in immunodeficient mice (4) and by the generation of induced pluripotent stem cells from DS 
fetal hematopoietic and TMD cells (23). Such experimental tools would allow systematic evaluation of human cT21 in the correct developmental context.

Whether DYRK1A is functioning through the NFAT pathway or another pathway in DS-AMKL, its inhibition may have therapeutic benefit. Malinge et al. provide a proof of concept that DYRK1A inhibitors may be clinically useful in the context of DS-AMKL by demonstrating that harmine, a small-molecule inhibitor of DYRK1A kinase activity, can inhibit the growth of megakaryoblastic leukemic cell lines with trisomy 21 (2). Thus, DYRK1A inhibitors may be a specific targeted therapy for DS-AMKL.

\section{Acknowledgments}

Our research of the leukemias of Down syndrome is funded by Children with Cancer (UK), NIH, Waxman Cancer Research Foundation (New York), Israel Cancer Research Foundation (New York), Israel Science Foundation (Regular, Legacy, and I-CORE programs), Israel USA Binational Foundation, the Chief Scientist of the Israeli Health Ministry, the Clinical Genetics Foundation (Israel), and William Lawrence Blanche Hughes Foundation (USA). Y. Birger is a European Hematology Association Fellow and funded by the Leukemia Research Foundation (Chicago).
Address correspondence to: Shai Izraeli, Sheba Medical Center, Tel Hashomer, 52621, Israel. Phone: 972.3.530.5943; Fax: 972.3.530.5942; E-mail: sizraeli@sheba. health.gov.il.

1. Hasle H. Pattern of malignant disorders in individuals with Down's syndrome. Lancet Oncol. 2001;2(7):429-436.

2. Malinge $S$, et al. Increased dosage of the chromosome 21 ortholog Dyrk1a promotes megakaryoblastic leukemia in a murine model of Down syndrome. J Clin Invest. 2012;122(3):948-962.

3. Malinge S, Izraeli S, Crispino JD. Insights into the manifestations, outcomes, and mechanisms of leukemogenesis in Down syndrome. Blood. 2009;113(12):2619-2628.

4. Chou ST, et al. Trisomy 21 enhances human fetal erythro-megakaryocytic development. Blood. 2008;112(12):4503-4506.

5. Tunstall-Pedoe $O$, et al. Abnormalities in the myeloid progenitor compartment in Down syndrome fetal liver precede acquisition of GATA1 mutations. Blood. 2008;112(12):4507-4511.

6. Izraeli S. Trisomy 21 tilts the balance. Blood. 2008; 112(12):4361-4362.

7. Hertzberg L, et al. Prediction of chromosomal aneuploidy from gene expression data. Genes Chromosomes Cancer. 2007;46(1):75-86.

8. De Vita S, et al. Trisomic dose of several chromosome 21 genes perturbs haematopoietic stem and progenitor cell differentiation in Down's syndrome. Oncogene. 2010;29(46):6102-6114.

9. Salek-Ardakani S, et al. ERG is a megakaryocytic oncogene. Cancer Res. 2009;69(11):4665-4673.

10. Stankiewicz MJ, Crispino JD. ETS2 and ERG promote megakaryopoiesis and synergize with alterations in GATA-1 to immortalize hematopoietic progenitor cells. Blood. 2009;113(14):3337-3347.

11. Ng AP, et al. Trisomy of Erg is required for myeloproliferation in a mouse model of Down syndrome. Blood. 2010;115(19):3966-3969.
12. Klusmann JH, et al. miR-125b-2 is a potential oncomiR on human chromosome 21 in megakaryoblastic leukemia. Genes Dev. 2010;24(5):478-490.

13. Park J, Song WJ, Chung KC. Function and regulation of Dyrk1A: towards understanding Down syndrome. Cell Mol Life Sci. 2009;66(20):3235-3240.

14. Arron JR, et al. NFAT dysregulation by increased dosage of DSCR1 and DYRK1A on chromosome 21. Nature. 2006;441(7093):595-600.

15. Muller MR, Rao A. NFAT, immunity and cancer: a transcription factor comes of age. Nat Rev Immunol. 2010;10(9):645-656.

16. Li X, et al. Calcineurin-NFAT signaling critically regulates early lineage specification in mouse embryonic stem cells and embryos. Cell Stem Cell. 2011;8(1):46-58.

17. Ram G, Chinen J. Infections and immunodeficiency in Down syndrome. Clin Exp Immunol. 2011; 164(1):9-16.

18. Lima FA, et al. Decreased AIRE expression and global thymic hypofunction in Down syndrome. J Immunol. 2011;187(6):3422-3430.

19. Baek KH, et al. Down's syndrome suppression of tumour growth and the role of the calcineurin inhibitor DSCR1. Nature. 2009;459(7250):1126-1130.

20. Giraudier S, et al. Overexpression of FKBP51 in idiopathic myelofibrosis regulates the growth factor independence of megakaryocyte progenitors. Blood. 2002;100(8):2932-2940.

21. Ge Y, et al. The role of the proto-oncogene ETS2 in acute megakaryocytic leukemia biology and therapy. Leukemia. 2008;22(3):521-529.

22. Ozery-Flato M, Linhart C, Trakhtenbrot L, Izraeli $\mathrm{S}$, Shamir R. Large-scale analysis of chromosomal aberrations in cancer karyotypes reveals two distinct paths to aneuploidy. Genome Biol. 2011;12(6):R61.

23. Chou ST, VanDorn D, Yao Y, French DL, Weiss MJ. 2011. Patient-derived induced pluripotent stem cells reveal distinct hematopoietic defects conferred by Trisomy 21 and truncated GATA- 1 . Abstract \#911 presented at: 53rd Annual American Society of Hematology Meeting. December 13, 2011. San Diego, California, USA.

\title{
Popeye proteins: muscle for the aging sinus node
}

\section{Bastiaan J. Boukens ${ }^{1,2}$ and Vincent M. Christoffels ${ }^{1,3}$}

\begin{abstract}
${ }^{1}$ Heart Failure Research Center, ${ }^{2}$ Department of Clinical and Experimental Cardiology, and ${ }^{3}$ Department of Anatomy, Embryology, and Physiology,
\end{abstract} Academic Medical Center, Amsterdam, The Netherlands

\begin{abstract}
The electrical impulses that dictate the rhythm of the heartbeat in normal situations and during exercise or stress are initiated by a small number of sinus node pacemaker cells. Senescence and dysfunction of the sinus node affects many people later in life, causing physiologically inappropriate heart rates, but the underlying mechanisms are not well understood. In this issue of the JCI, Froese and colleagues show that deficiency in either Popeye domain containing 1 (Popdc1) or Popdc2 leads to sinus node dysfunction under stressed conditions in aged mice. The mechanism reported to underlie the effects of Popdc1/2 deficiency in mice may cause the stress-induced sinus node dysfunction found in many aged individuals and may point to new strategies for therapeutic intervention.
\end{abstract}

Conflict of interest: The authors have declared that no conflict of interest exists.

Citation for this article: J Clin Invest. 2012;

122(3):810-813. doi:10.1172/JCI62588.
The rhythmic forward flow of blood through the major arterial vessels is controlled by coordinated electrical activation of the heart. Electrical activation in the heart originates in the sinus node, which is located in the right atrium near the entrance of the superior vena cava (1). Sinus node cells differ from normal atrial and ventricular cardiomyocytes in many respects. They develop, during embryogenesis, from precursor cells distinct from those that give rise to cardiomyocytes in the remainder of the heart. Moreover, throughout life, they maintain a molecular program that is unique and quantitatively different from that of normal atrial and ventricular cardiomyocytes (2). This molecular program endows them with unique characteristics: for example, unlike atrial and ventricular cardiomyocytes, sinus node 